\title{
O uso da estreptoquinase no tratamento da oclusão arterial aguda pós-cateterização da artéria femoral em crianças com menos de $10 \mathrm{~kg}$
}

\author{
The use of streptokinase in the treatment of acute arterial occlusion \\ after catheterization of the femoral artery in children \\ weighing less than $10 \mathrm{~kg}$
}

\section{Nilo César Barbosa Mandelli' ${ }^{1}$, Cláudio Nhuch ${ }^{2}$, Paulo Roberto Fontes ${ }^{3}$, Haroldo Diez Paiva ${ }^{4}$, Raul Rossi ${ }^{5}$, Mario Frederico Chagas Pereira ${ }^{2}$, Silvio Perini²}

\section{Resumo}

Contexto: $\mathrm{O}$ tratamento da oclusão arterial aguda em menores de $5 \mathrm{~kg}$ tem constituído tema de discussão.

Objetivos: Avaliar o tratamento do quadro da oclusão arterial aguda pós-cateterismo da artéria femoral em crianças com menos de $10 \mathrm{~kg}$ com o uso de heparina isolada e também associada com estreptoquinase, e comparar os resultados do exame físico (como diagnóstico), da reversão da oclusão arterial, de complicações e de exames laboratoriais nos dois métodos.

Métodos: Trinta casos de oclusão da artéria femoral foram identificados em 1.583 cateterismos em crianças no Instituto de Cardiologia de Porto Alegre, entre 1992 e 2000. Os pacientes foram divididos em dois grupos: um usou apenas heparina (14 casos), e o outro usou heparina associada com estreptoquinase (16 casos). Os exames laboratoriais (tempo de protrombina, tempo de tromboplastina parcial ativado e fibrinogênio) coletados antes e durante a infusão intravenosa foram avaliados estatisticamente, assim como o tempo de uso da medicação, as complicações e os resultados.

Resultados: O exame físico mostrou-se método fidedigno para avaliar a oclusão; no grupo que utilizou a associação de heparina e estreptoquinase, houve a resolução de $87 \%$ dos casos de oclusão arterial, e a principal complicação foi sangramento no sítio de punção em $56,3 \%$ dos pacientes. Os resultados apresentaram $\mathrm{p}<0,05$. Os exames laboratoriais não tiveram significado estatístico.

Conclusão: A estreptoquinase associada com a heparina é mais efetiva do que a heparina isolada no tratamento da oclusão arterial aguda da artéria femoral pós-cateterismo, tanto que sua associação apresenta uma redução do risco relativo de $88 \%$ em relação à heparina isolada.

Palavras-chave: Estreptoquinase, criança, cateterismo, oclusão arterial.

\begin{abstract}
Background: The treatment of acute arterial occlusion in children weighing less than $5 \mathrm{~kg}$ has been widely discussed.

Objectives: To evaluate the treatment of acute arterial occlusion after catheterization of the femoral artery in children weighing less than $10 \mathrm{~kg}$ using heparin alone and associated with streptokinase, and to compare the results of physical examination (such as diagnosis), reversal of the arterial occlusion, complications and laboratory tests between both methods.

Methods: Thirty cases of femoral artery occlusion were identified among 1,583 catheterizations in children at Instituto de Cardiologia de Porto Alegre between 1992 and 2000. The patients were divided into two groups: one used heparin alone ( 14 cases) and the other used heparin associated with streptokinase (16 cases). The laboratory tests (prothrombin time, activated partial thromboplastin time and fibrinogen) performed before and during the intravenous infusion were statistically analyzed, as well as period of drug administration, complications and results.
\end{abstract}

Results: Physical examination proved to be reliable to evaluate occlusion; in the group using heparin associated with streptokinase, there was resolution of the arterial occlusion in $87 \%$ of cases, and the main complication was bleeding at the puncture site, which was present in $56.3 \%$ of the patients. These results showed $\mathrm{p}<0.05$. Laboratory tests were not statistically significant.

Conclusion: Streptokinase associated with heparin is more effective on acute artery occlusions following femoral catheterization than heparin alone. Its association presents an $88 \%$ rate of relative risk reduction in relation to heparin alone.

Keywords: Streptokinase, child, catheterization, arterial occlusion.

1. Cirurgião vascular, Instituto de Cardiologia - Fundação Universitária de Cardiologia (IC-FUC), Porto Alegre, RS. Mestre em Medicina, Fundação Faculdade Federal de Ciências Médicas de Porto Alegre (FFFCMPA), Porto Alegre, RS.

2. Cirurgião vascular, IC-FUC, Porto Alegre, RS.

3. Doutor. Professor adjunto, Disciplina de Cirurgia, FFFCMPA, Porto Alegre, RS.

4. Professor titular, Disciplina de Cirurgia Vascular, FFFCMPA, Porto Alegre, RS.

5. Medico hemodinamicista infantil, IC-FUC, Porto Alegre, RS.

Este estudo foi realizado no IC-FUC, Porto Alegre, RS. É baseado em tese de mestrado (Clínica Cirúrgica) desenvolvida na FFFCMPA em 2002 e foi apresentado no XXIII Congresso de Cardiologia do Rio Grande do Sul, em 2003, e no 36ํㅡㄹ Congresso Brasileiro de Cirurgia Vascular, em 2005.

Artigo submetido em 24.07.06, aceito em 18.01.07.

J Vasc Bras 2007;6(1):42-49.

Copyright $\odot 2007$ by Sociedade Brasileira de Angiologia e de Cirurgia Vascular 


\section{Introdução}

A oclusão arterial aguda dos membros superiores e inferiores nas crianças, iatrogênica ou não, raramente leva à perda dos membros $(2,5 \%)^{1,2}$. Porém, freqüentemente evolui para déficit de crescimento (Figura 1$)^{3-5}$. O tratamento de eleição nas oclusões arteriais agudas por trombose secundária, causada pelo cateter ou lesão da parede arterial, é a cirurgia; entretanto, os resultados não são satisfatórios em crianças, devido ao reduzido calibre dos vasos ${ }^{6-10}$. Por esse motivo, foi iniciada a utilização de medicamentos trombolíticos e anticoagulantes, por via sistêmica ou injeção direta intra-arteri$\mathrm{al}^{5,8,11}$.

O tratamento tardio das complicações apresentadas pela oclusão tornou-se um desafio. O desvio de coluna decorrente da diferença de comprimento das pernas é normalmente corrigido por palmilhas, botas ou cirurgias ortopédicas ${ }^{12}$. A reconstrução arterial é complexa, pois próteses não acompanham o crescimento da criança, as veias ou artérias apresentam diâmetro reduzido.

Sabe-se que os sistemas de hemostasia e fibrinólise nas crianças são imaturos e se desenvolvem após os 6 meses de idade. Quando se traça um paralelo entre os níveis de fatores pró e anticoagulantes, tende a ocorrer uma leve tendência à coagulação, sendo maior a predisposição em crianças prematuras e com doenças associadas (policitemia, insuficiência respiratória, renal ou hepática $)^{13}$.

A causa iatrogênica mais freqüente é o cateterismo da artéria femoral pela técnica de Seldinger ${ }^{14-17}$, com o intuito de realizar estudo hemodinâmico. A incidência em pacientes com menos de $10 \mathrm{~kg}$ (do nascimento até 2 anos) é de 0,8-9\%, tendo diminuído após a introdução da heparinização sistêmica a partir de $1970^{5,18-25}$. Fatores como peso da criança menor que $10 \mathrm{~kg}$, maior diâmetro do cateter e maior duração do procedimento estão relacionados com maior incidência de complicações. Outra causa de oclusão arterial é o cateterismo da artéria umbilical levando à trombose da aorta abdominal e veia umbilical levando à trombose do átrio direito $^{21,26}$.

As drogas utilizadas mais freqüentemente são a heparina, a estreptoquinase, a uroquinase e o ativador do plasminogênio tecidual (TPA) ${ }^{15,27-32}$.

Os objetivos deste trabalho foram: 1) comparar o uso isolado da heparina com a sua associação à estreptoquinase nas oclusões arteriais agudas em crianças com menos de $10 \mathrm{~kg} ; 2$ ) estudar as complicações decorrentes dos métodos; 3 ) avaliar as alterações laboratoriais - tempo de protrombina (TP), tempo de tromboplastina parcial ativado (TTPA) e fibrinogênio na ocorrência de complicações; 4) verificar se o exame físico é um método diagnóstico fidedigno para a confirmação de oclusão arterial aguda pós-cateterismo.

\section{Métodos}

Foram avaliados 1.583 cateterismos da artéria femoral comum, realizados no Instituto de Cardiologia de Porto Alegre, para diagnóstico ou tratamento de patologias congênitas do miocárdio ou da aorta torácica, em crianças abaixo de $10 \mathrm{~kg}$, sem distinção de sexo ou raça. Foram diagnosticados 30 casos de oclusão arterial aguda, sendo que estes foram separados em dois grupos:

- Grupo $1(\mathrm{GH})$ - pacientes que usaram somente a heparina endovenosa (veia periférica ou central) na dose de 25 unidades $/ \mathrm{kg} / \mathrm{h}$, sendo o estudo realizado de modo retrospectivo ( $1^{\circ}$ de março de 1992 a 23 de outubro de 1996), tendo um total de 14 casos;

- Grupo 2 (GHE) - pacientes que usaram heparina por via endovenosa na dose de $25 \mathrm{U} / \mathrm{kg} / \mathrm{h}$ e estreptoquinase na dose de $3.000 \mathrm{U} / \mathrm{kg} / \mathrm{h}$, em um total de 16 casos, sendo o estudo realizado prospectivamente $\left(1^{\circ}\right.$ de novembro de 1996 a 31 de abril de 2000). O delineamento do trabalho foi de estudo clínico quase experimental.

As medicações foram utilizadas até o reaparecimento dos pulsos. O limite para uso da medicação no $\mathrm{GH}$, caso o pulso não tivesse retornado, foi estabelecido como a melhora da perfusão da extremidade (volta da coloração e aquecimento do pé, comparado com o 


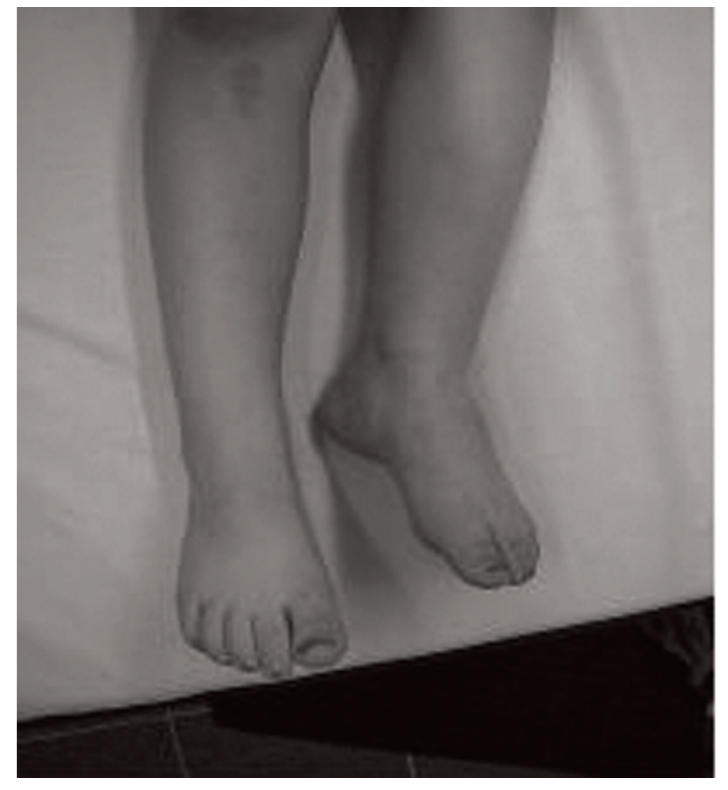

Figura 1 - Criança de 3 anos com déficit de crescimento

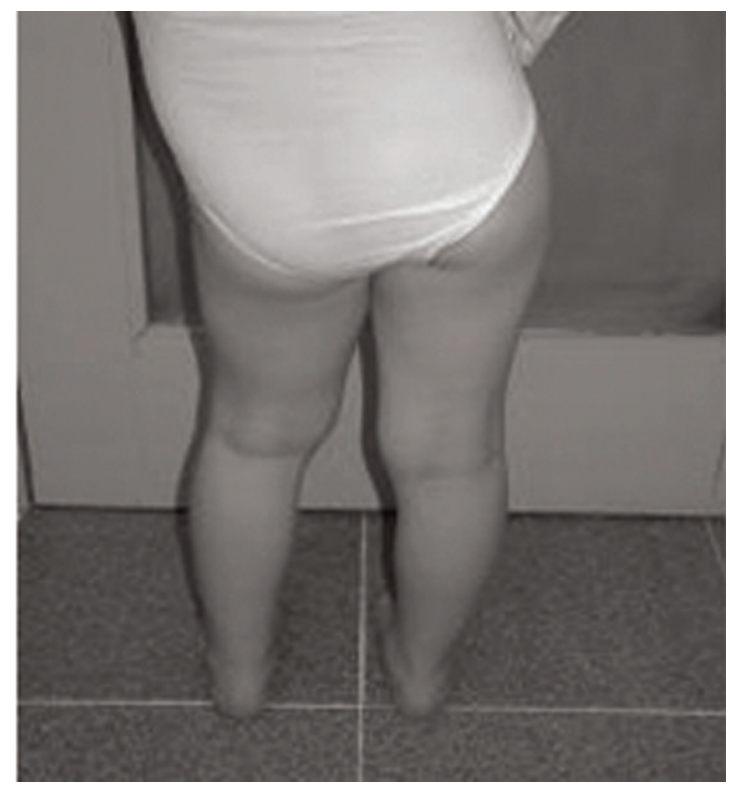

membro superior ou contralateral, se sem lesão). Já no GHE, a medicação foi mantida por 48 horas, caso o pulso não tivesse retornado, independente da perfusão do pé.

As variáveis sexo, peso, idade, tempo decorrido até o atendimento pós-cateterismo, sinais do Doppler audível pré e pós-término do procedimento (GHE), sinais e sintomas clínicos, dose utilizada de medicação, tempo de uso da medicação e complicações pós-uso das medicações foram avaliadas. Todos os pacientes receberam, por via endovenosa (periférica ou central), dose única de heparina $25 \mathrm{U} / \mathrm{kg}$, dada antes de iniciar cateterismo. Sinais positivos de oclusão arterial foram: ausência de pulso na artéria em que se realizou cateterismo e distalmente a ela, palidez do pé e cianose não-fixa sem edema. $\mathrm{O}$ Doppler era positivo quando apresentava som audível semelhante aos sons dos locais de pulso normal. Todos os pacientes, após suspeita diagnóstica da oclusão, usaram heparina, por 6 horas, na dose de $25 \mathrm{U} / \mathrm{kg} / \mathrm{h}$, para excluir o vasoespasmo pós-exame. O tempo de diagnóstico de oclusão arterial foi o tempo decorrido desde a hora em que terminou o procedimento até a hora da chegada do cirurgião vascular. Foram coletados TP, TTPA e plasminogênio antes do início da infusão. As complicações foram classificadas em:

1) sangramento no local da punção;

2) sangramento em outros locais: cérebro, abdômen, tórax e ocular;

3) reações alérgicas;

4) hipotensão com choque causado por sangramento ou anafilaxia.

Foram critérios contra-indicativos para uso de heparina e estreptoquinase: presença de doença congênita cerebral com risco de sangramento, sangramento ativo determinado ou história de anafilaxia à medicação. Para analise das variáveis peso, dose de heparina, TP pré e pós-medicação, TTPA pré e pós-medicação, fibrinogênio pré e pós-medicação, foi utilizado o teste $t$ de Student; para as variáveis idade, tempo decorrido até a confirmação do diagnóstico definitivo pelo cirurgião vascular e tempo de uso de medicação, foi usado o teste de Mann-Whitney; para a variável raça e na comparação das complicações, usou-se o teste exato de Fisher; para a variável sexo e na comparação dos resultados, usou-se a continuity correction ${ }^{33}$. 


\section{Resultados}

Dos 1.583 cateterismos $(\mathrm{GH}, \mathrm{n}=628$; GHE, $\mathrm{n}=$ 955), $30(1,89 \%)$ resultaram em oclusão da artéria femoral (GH, $\mathrm{n}=14,2,22 \%$; GHE, $\mathrm{n}=16,1,67 \%)$.

A Tabela 1 mostra que não houve diferença estatística entre os dois grupos quanto ao sexo, raça, idade, peso, tempo diagnóstico e dose de heparina. Ocorreu diferença estatística no tempo de utilização da medicação. Não ocorreram complicações no GH. No GHE, nove pacientes $(56,25 \%)$ tiveram sangramento no local da punção femoral, resolvido somente com compressão prolongada (em média 16 minutos, sem hipotensão) sobre o local. Apenas um paciente apresentou fibrinogênio abaixo do normal ( $90 \mathrm{mg} / \%$ ) no GHE, sendo que o mesmo não apresentou sangramento, mostrando que exames não estavam relacionados com risco de sangramento.

Todos os pacientes que tiveram oclusão não tinham pulso pós-cateterismo, e Doppler audível estava ausente ou com som monofásico nas artérias distais à punção (femoral superficial, poplítea, tibial anterior e posterior). Com a melhora clínica, houve reaparecimento do pulso e sinal audível do Doppler igual ao membro não-ocluído.
O efeito do tratamento é visto na Tabela $1 . \mathrm{O}$ risco de permanecer ocluído no GHE foi de $12,5 \%$, e no GH, de $100 \%$, com um risco relativo de 0,125 (IC95\% 0,03-0,46) e redução absoluta de risco (RAR) de 87,5\%. O uso de heparina + estreptoquinase trouxe uma redução em $88 \%$ das oclusões arteriais agudas pós-cateterismo femoral em crianças quando comparado com o uso de heparina pura. Isso mostra uma redução de risco relativo (RRR) igual a 88\% (IC95\% 54-97\%). Quanto ao numero necessário para tratamento (NNT), este foi de 1,1 (IC95\% 0,9-1,2), ou seja, aproximadamente, para cada paciente tratado, evita-se uma oclusão.

\section{Discussão}

No GH, o estudo foi realizado retrospectivamente, pois não seria eticamente aceitável novo estudo, uma vez que não ocorreu resolução do quadro em nenhum paciente (todos com déficit de crescimento).

Sabe-se que o tratamento mais eficaz das oclusões arteriais agudas de origem não-aterosclerótica é a intervenção cirúrgica direta e imediata sobre a artéria, com a realização da correção da lesão ou a retirada do trombo que nela se alojou ${ }^{1,34}$. Alguns autores utilizaram fibrinolíticos, em altas doses, por via endovenosa ${ }^{29,35-38}$, ou em baixas doses via intra-arterial, com auxílio de

Tabela 1 - Estratificação dos pacientes quanto aos grupos

\begin{tabular}{lccc}
\hline Variável & $\begin{array}{c}\text { Grupo heparina } \\
\mathbf{n}=\mathbf{1 4}\end{array}$ & $\begin{array}{c}\text { Grupo heparina + estreptocinase } \\
\mathbf{n}=\mathbf{1 6}\end{array}$ & $\mathbf{p}$ \\
\hline Cor da pele & & & \\
Branca & $12(85,7 \%)$ & $16(100 \%)$ & 0,2092 \\
Preta & $2(14,3 \%)$ & $0(0 \%)$ & \\
Sexo & & & 0,98049 \\
Masculino & $6(42,9 \%)$ & $8(50 \%)$ & \\
Feminino & $8(57,1 \%)$ & $8(50 \%)$ & $\cong 0,9999$ \\
Idade (dias) & $52(26-180)$ & $45(29-180)$ & 0,516 \\
Peso (kg) & $4,58 \pm 1,59$ & $5,06 \pm 2,24$ &
\end{tabular}


cateteres, resolvendo a oclusão aguda. No caso das crianças, a intervenção cirúrgica pode piorar a lesão da árvore arterial, já que o diâmetro dessas artérias é muito pequeno, principalmente nas com menos de 10 $\mathrm{kg}^{5,7,8,11}$; e com o uso de cateteres, para injeção intratrombo, há o risco de a nova punção causar lesão em outra artéria.

A eficácia da heparina isolada no tratamento da oclusão arterial foi relatada por $\mathrm{Wessel}^{5}$, onde resolveu $50 \%$ dos casos de oclusão arterial aguda nas crianças. No presente trabalho, isso não foi comprovado. A crítica que se pode fazer à publicação é o fato de que vasoespasmo arterial pós-exame não foi avaliado pelos autores $^{39}$.

Todos os pacientes tiveram sinais clínicos (palidez do pé e cianose não-fixa sem edema) presentes. Os sintomas clínicos foram de difícil definição, devido à dificuldade de comunicação com as crianças. Isso mostra que os sinais ajudam no diagnóstico, mas que os sintomas não contribuem para o mesmo ${ }^{40,41}$.

O Doppler foi utilizado somente nos pacientes do GHE como método para confirmar o diagnóstico da oclusão arterial aguda e quando da avaliação da resposta do tratamento feita através do exame físico vascular do paciente. Esse método diagnóstico mostrou que o exame físico pode ser $100 \%$ sensível, sendo, assim, suficientemente fidedigno para avaliação no pré e póscateterismo. Sabe-se que, quando o Doppler é positivo e não há pulso arterial, o som escutado com o Doppler é do fluxo que está chegando à artéria ocluída pelas artérias colaterais, o que já foi comprovado por Hurowitz $^{2}$, no exame arteriográfico, e também confirmado por Deeg, com utilização do exame de eco-Doppler.

O tempo de uso da heparina e da estreptoquinase não está definido pela literatura. Os trabalhos que usam heparina não se referem ao tempo, pois sua ação não é direta sobre o trombo ${ }^{5}$, e os da estreptoquinase têm como exemplo o trabalho de Kirk \& Qureshi ${ }^{42}$, que usam-na por 48 horas; no entanto, Saxena ${ }^{21}$ relata seu uso por mais de 48 horas. Kothari ${ }^{43}$ relaciona tempo aumentado ou ineficácia com os baixos níveis de fibrinogênio; há relatos de que a criança tem em torno de 50 a $70 \%$ da quantidade do fibrinogênio e plasminogênio do adulto ${ }^{19,28,44-49}$. Corrigam ${ }^{44,50}$ demonstra que a criança necessita de uma concentração 11 vezes maior de estreptoquinase ou uroquinase e cinco vezes a concentração de TPA do adulto para sua ação. Já Brus $^{18}$, quando não obteve o efeito desejado no período de 48 horas, infundiu plasma fresco (fornecendo plasminogênio) e repetiu a dose de estreptocinase por mais 48 horas, obtendo, assim, quase 100\% de eficácia.

O tempo médio de uso da estreptoquinase neste estudo foi de 4 horas e 15 minutos, e o tempo máximo foi de 48 horas. Alguns trabalhos da literatura adotam um tempo maior de uso para obtenção do efeito positivo. Prichard $^{51}$ utilizou 44 horas, Ino $^{7}, 48$ horas, Kirk \& Qureshi $^{42}$, um limite máximo de 44 horas, Brus ${ }^{18}, 120$ horas, Kothari ${ }^{43}$, 48 horas, e Saxena ${ }^{21}$ demonstraram uma diminuição do tempo, em relação aos trabalhos anteriores, de 5 horas. Já em relação ao tempo de uso da heparina, no $\mathrm{GH}$, ele foi de 88 horas, e o término da infusão ocorreu quando se obteve a melhora da perfusão e não o retorno do pulso, motivo pelo qual apresentou um tempo alto de uso da medicação.

A dose de heparina, na literatura ${ }^{21}$, varia de $10 \mathrm{U} / \mathrm{kg} / \mathrm{h}$ a $25 \mathrm{U} / \mathrm{kg} / \mathrm{h}$, usada de forma contínua. No GHE, ela foi utilizada associada com estreptoquinase, com o objetivo de evitar a retrombose ${ }^{43}$, no caso de lesão do endotélio da artéria pelo coágulo junto à parede ou pela própria punção. Por esse motivo, foi mantida por 48 horas após o retorno do pulso e suspensão da estreptoquinase, pois as plaquetas, nesse período de tempo, cobririam essa área lesada, evitando a formação de trombo ${ }^{52}$. Alguns autores usam o ácido acetilsalicílico, na dose de $10 \mathrm{mg} / \mathrm{kg}$, em vez de heparina após a suspensão da estreptoquinase. A dose da estreptoquinase, na literatura, varia de $1.000 \mathrm{U} / \mathrm{kg} / \mathrm{h}$ até $4.000 \mathrm{U} / \mathrm{kg} / \mathrm{h}$, não mostrando relação dose/complicação de sangramento ${ }^{43}$. Usamos a dose de $3.000 \mathrm{U} / \mathrm{kg} / \mathrm{h}$ por via endovenosa contínua, conforme Kothari ${ }^{43}$.

Nos GH e GHE, todos os pacientes estudados apresentaram valores de TTPA alterados, acima do normal, resultado este colhido antes do uso da medicação, visto que todos eles haviam usado heparina du- 
rante o cateterismo da artéria femoral e, no caso de suspeita de oclusão, mantiveram-na por mais 6 horas, para descartar diagnóstico de vasoespasmo pela punção arterial ${ }^{39}$. Não se pode esquecer que há relação direta entre as duas vias da coagulação, onde a atividade da via extrínseca é medida através do TP e pode se encontrar, às vezes, um pouco alterada com o uso da heparina ${ }^{53}$.

O TP e o TTPA são ineficazes para acompanhar a ação da estreptoquinase ${ }^{21,54}$. A dosagem do fibrinogênio pode ser útil, mas não nos dá o prognóstico quanto à chance de ocorrer sangramento ${ }^{54}$. Todos os pacientes que apresentaram sangramento tinham alteração do TP e do TTPA, conforme explicação anterior, e valores normais para fibrinogênio. Na literatura, Prichard $^{51}$ faz menção à dificuldade de saber se haveria sangramento com os exames laboratoriais citados acima, e Kothari ${ }^{43}$ relata a dificuldade de se estabelecer uma relação entre os valores dos exames laboratoriais e o risco de sangramento, mesmo tendo associado a eles a dosagem de produtos da degradação da fibrina.

O ponto importante com relação ao uso das medicações fibrinolíticas são suas complicações ${ }^{7,55-58}$. Viuse, acima, que as complicações decorrentes do uso da estreptoquinase são difíceis de ser previstas mediante avaliação laboratorial do paciente no transcorrer da sua infusão endovenosa. Em vista desse problema, o uso da medicação poderia ser contra-indicado. No entanto, no presente trabalho, as complicações foram de pequena magnitude. A única complicação registrada foi sangramento no local da punção arterial, em 56,25\% dos casos, o qual foi resolvido facilmente mediante a compressão do local da punção e suspensão da medicação em infusão. Na literatura, são citados outros lugares com risco de sangramento na infância, como casos de hemorragia cerebral e retroperitonial e nos sistemas urinário e digestivo ${ }^{59}$; no entanto, os trabalhos com uso de estreptoquinase para as oclusões pós-cateterismo não relatam casos de sangramento em outros locais além do local de punção ${ }^{18,21}$. Uma complicação grave como a hemorragia cerebral, conforme revisão de 30 anos feita por Zenz ${ }^{59}$, é considerada muito rara, ficando em torno de $0,4 \%$ em crianças de até 1 mês de vida. Outra complicação como a anafilaxia não é encontrada em crianças; os sangramentos digestivos somente foram relatados nos pacientes que utilizaram estreptoquinase no tratamento da trombose de veia renal. Cabe lembrar que a insuficiência renal e a insuficiência hepática, quando presentes e se associadas à estreptoquinase, aumentam a chance de ocorrer sangramento ${ }^{59}$.

Quanto ao desfecho do trabalho, os resultados foram semelhantes aos da literatura, onde Brus ${ }^{18}$ teve $85 \%$ de resolução, Kothari ${ }^{43}, 100 \%$, e Saxena ${ }^{28}$, $95 \%$. Os resultados obtidos foram estatisticamente significativos em relação à resolução das oclusões e ao tempo de demora para que isso ocorra, tendo-se obtido RAR de $87,5 \%$, RRR de $88 \%$ e NNT de 1,1. Conclui-se que a associação da estreptoquinase com heparina, no tratamento das oclusões arteriais agudas pós-cateterismo em crianças abaixo de $10 \mathrm{~kg}$, empregando-se a metodologia acima descrita, resolve as oclusões arteriais em 87,5\% dos casos. As complicações ocorridas com o uso da estreptoquinase associada com heparina são consideradas não-graves, e os exames laboratoriais colhidos nas crianças não demonstraram auxiliar na prevenção do sangramento. No transcorrer da infusão endovenosa de estreptoquinase, o exame físico é método fidedigno para confirmação do diagnóstico da oclusão arterial aguda nas crianças pós-cateterismo.

\section{Referências}

1. Haimovici H. Arterial embolism of the extremities and technique of embolectomy. In: Haimovici H, Ascer E, Hollier LH, Strandness Jr. DE, Towne JB. Vascular surgery: principles and techniques. 4th ed. Massachusetts: Blackwell Science; 1996. p. 423-44.

2. Hurwitz RA, Franken EA Jr., Girod DA, Smith JA, Smith WL. Angiographic determination of arterial patency after percutaneous catheterization in infants and small children. Circulation. 1977;56:102-5.

3. Fairbairn JF. Clinical manifestations of perpheral vascular disease. Juergens JL, Spittell JR, Fairbairn JF II. Peripheral vascular disease. 5th ed. Philadelphia: WB Saunders; 1980. p. 3.

4. Burrows PE, Benson LN, Williams WG, et al. Iliofemoral arterial complications of balloon angioplasty for systemic obstructions in infants and children. Circulation. 1990;82:1697-704.

5. Wessel DL, Keane JF, Fellows KE, Robichaud H, Lock JE. Fibrinolytic therapy for femoral arterial thrombosis after cardiac catheterization in infants and children. Am J Cardiol. 1986;58:347-51. 
6. Fogarty TJ. Rutherford RB. Vascular surgery. 4th ed. Philadelphia: WB Saunders; 1995. p. 410-4.

7. Ino T, Benson LN, Freedom RM, Barker GA, Airpursky A, Rowe RD. Thrombolytic therapy for femoral artery thrombosis after pediatric cardiac catheterization. Am Heart J. 1988;115:633-9.

8. Stanger P, Heymann MA, Tarnoff H, Hoffman JI, Rudolph AM. Complications of cardiac catheterization of neonates, infants, and children. A three-year study. Circulation. 1974;50:595-608.

9. Mansfield PB, Gazzaniga AB, Litwin SB. Management of arterial injuries related to cardiac catheterization in children and young adults. Circulation. 1970;42:501-7.

10. Mason AC, Llull R, Bentz ML. Revascularization of the upper extremity in a preterm infant: a case report and review of the literature. Ann Plast Surg. 1999;43:646-8

11. While JS, Talbert JL, Haller JA Jr. Peripheral arterial injuries in infants and children. Ann Surg. 1968;167:757-67.

12. Beaty JH. Congenital anomalies of lower extremity. In: Crenshaw AH. Campbell 's operative orthopedics. 7th ed. St. Louis: Mosby; 1987. p. 2623-780.

13. Corrigan JJ Jr., Sleeth JJ, Jeter M, Lox CD. Newborn's fibrinolytic mechanism: components and plasmin generation. Am J Hematol. 1989;32:273-8.

14. Seldinger SI. Catheter replacement of the needle in percutaneous arteriography; a new technique. Acta Radiol. 1953;39:368-76.

15. Tokel K, Yildirim SV, Varan B, Ekici E. Sequential balloon dilatation for combined aortic valvular stenosis and coarctation of the aorta in a single catheterization procedure: a prognostic evaluation based on long-term follow up. J Invasive Cardiol. 2006;18:65-9.

16. Galal MO, Schmaltz AA, Joufan M, Benson L, Samatou L, Halees Z. Balloon dilation of native aortic coarctation in infancy. Z Kardiol. 2003;92:735-41.

17. Saxena A, Gupta R, Kumar RK, Kothari SS, Wasir HS. Predictors of arterial thrombosis after diagnostic cardiac catheterization in infants and children randomized to two heparin dosages. Cathet Cardiovasc Diagn. 1997;41:400-3.

18. Brus F, Witsenburg M, Hofhuis WJ, Hazelzet JA, Hess J. Streptokinase treatment for femoral artery thrombosis after arterial cardiac catheterisation in infants and children. $\mathrm{Br}$ Heart J. 1990;63:291-4.

19. Gamba P, Pettenazzo A, Kalapurackal M, Saia SO, Guglielmi M. Primary occlusion of the iliac and femoral artery in two newborn infants: efficacy of medical treatment. J Pediatr Surg. 1993;28:735-7.

20. Kothari SS, Kumar RK, Varma S, Saxena A. Thrombolytic therapy in infants for femoral artery thrombosis following cardiac catheterization. Indian Heart J. 1996;48:246-8.

21. Saxena A, Gupta R, Kumar RK, Kothari SS, Wasir HS. Predictors of arterial thrombosis after diagnostic cardiac catheterization in infants and children randomized in two heparin dosages. Cathet Cardiovasc Diagn. 1997;41:400-3.

22. Richardson R, Applebaum H, Touran T, et al. Effective thrombolytic therapy of aortic thrombosis in the small premature infant. J Pediatr Surg. 1988;23:1198-200.
23. Strife JL, Ball WS Jr., Towbin R, Keller MS, Dillon T. Arterial occlusions in neonates: use of fibrinolytic therapy. Radiology. 1988;166:395-400.

24. Aydogan U. Arterial duct closure with detachable coils: application in the small child. Asian Cardiovasc Thorac Ann. 2002;10:124-8.

25. Agnoletti G, Bonnet C, Boudjemline Y, et al. Complications of pediatric interventional catheterisation: an analysis of risk factors. Cardiol Young. 2005; 15:402-8.

26. Ross P Jr., Ehrenkranz R, Kleinman CS, Seashore JH. Thrombus associated with central venous catheters in infants and children. J Pediatr Surg. 1989;24:253-6.

27. O'Relly RA. Anticoagulants, antithrombotics and thrombolytics drugs. In: Gilman AG, Goodman LS, Rall TW, Murad F. The pharmacological basis of therapeutics. 7 th ed. Macmillan; 1987. p. 878-90.

28. Verstraete M, Vermylen J, Amery A, Vermylen C. Thrombolytic therapy with streptokinase using a standard dosage scheme. Br Med J. 1966;1:454-6.

29. Deucher GP, Mickelberg A, Nunes P, Cabral CA, Degni M. Nossa experiência clínica com a fibrinoliticoterapia pela estreptocinase nas embolias e tromboses arteriais. Rev Bras Cardiovasc. 1975;11:112-7.

30. Albisetti M, Schmugge M, Haas R, et al. Arterial thromboembolic complications in critically ill children. J Crit Care. 2005;20:296-300.

31. The Thrombolysis in Myocardial Infarction (TIMI) trial. Phase I findings. TIMI Study Group. N Engl J Med. 1985;312:932-6.

32. Van de Werf F, Ludbrook PA, Bergmann SR, et al. Coronary thrombolysis with tissue-type plasminogen activator in patients with evolving myocardial infarction. N Engl J Med. 1984;310:609-13.

33. Fletcher RH, Fletcher SW, Wagner EH. Tratamento. In: Fletcher RH, Fletcher SW, Wagner EH. Epidemiologia clínica: elementos essenciais. $3^{\mathrm{a}}$. ed. Porto Alegre: Artmed; 1996. p. 145-73.

34. Haimovici H. Acute arterial thrombosis. In: Haimovici $H$, Ascer E, Hollier LH, Strandness Jr. DE, Towne JB. Vascular surgery: principles and techniques. 4th ed. Massachusetts: Blackwell; 1996. p. 458-65.

35. Ramos AI, Ramos RF, Togna DJ, et al. Fibrinolytic therapy for thrombosis in cardiac valvular prosthesis short and long term results. Arq Bras Cardiol. 2003;81:393-8, 387-92. Epub 2003 Nov 5.

36. Gupta D, Kothari SS, Bahl VK, et al. Thrombolytic therapy for prosthetic valve thrombosis: short-and long-term results. Am Heart J. 2000;140:906-16.

37. Bolz D, Kuhne T, Jivraj R, Hammer J. Thrombolysis of prosthetic tricuspid valve thrombosis with human recombinant tissue plasminogen activator in an adolescent. Pediatr Cardiol. 2000;21:397-400.

38. Atiyeh BS, Hashim HA, Hamdan AM, Musharafieh RS. Local recombinant tissue plasminogen activator (rt-PA) thrombolytic therapy in microvascular surgery. Microsurgery. 1999;19:265-71. 
39. Bergstrom K, Jorulf $\mathrm{H}$. Reaction of femoral and common carotid arteries in infants after puncture or percutaneous catheterization. Acta Radiol Diagn (Stockh). 1976;17:577-80.

40. Pousti TJ, Wilson SE, Willams RA. Clinical examination of the vascular system. Veith FJ, Hobson RW, Williams RA, Wilson SE. Vascular surgery: principles and practice. 2nd ed. New York: McGraw-Hill; 1994. p. 74-89.

41. Ascer E, Gennaro M, Mohan Chittur, Veith FJ. Management of acute lower-extremety ischemia. In: Callow AD, Ernst CB. Vascular surgery: theory and practice. Connecticut: Appleton \& Lange; 1995. p. 715-34.

42. Kirk CR, Qureshi SA. Streptokinase in management of arterial thrombosis in infancy. Int J Cardiol. 1989;25:15-20.

43. Kothari SS, Varma S, Wasir HS. Thrombolytic therapy in infants and children. Am Heart J. 1994;127:651-7.

44. Corrigan JJ Jr., Sleeth JJ, Jeter M, Lox CD. Newborn's fibrinolytic mechanism: components and plasmin generation. Am J Hematol. 1989;32:273-8.

45. Peters M, ten Cate JW, Jansen E, Breederveld C. Coagulation and fibrinolytic factors in the first week of life in healthy infants. J Pediatr. 1985;106:292-5.

46. Peters M, ten Cate JW, Koo LH, Breederveld C. Persistent antithrombin III deficiency: risk factor for thromboembolic complications in neonates small for gestacional age. J Pediatr. 1984;105:310-4.

47. Vergnes C, Larnaudie B, Hourdille P, Roudaut MF, Choussat A, Boisseau MR. Thrombose aortique abdominale sous-renale neonatale. Presse Med. 1985;14:492-9.

48. Batsford S, Brundiers M, Schweier O, Horbach E, Monting JS. Antibody to streptococcal cysteine proteinase as a seromarker of group A Streptococcal (Streptococcus pyogenes) infections. Scand J Infect Dis. 2002;34:407-12.

49. Nowak-Gottl U, Auberger K, Halimeh S, et al. Thrombolysis in newborns and infants. Thromb Haemost. 1999;82 Suppl 1:112-6

50. Vojdani A, Bazargan M, Vojdani E, et al. Heat shock protein and gliadin peptide promote development of peptidase antibodies in children with autism and patients with autoimmune disease. Clin Diagn Lab Immunol. 2004; 11:515-24.
51. Pritchard SL, Culham JA, Rogers PC. Low-dose fibrinolytic therapy in infants. J Pediatr. 1985;106:594-8.

52. Silver D, Kikta MJ. Thrombogenesis and thrombolysis. In: Haimovici H, Ascer E, Hollier LH, Strandness Jr. DE, Towne JB. Vascular surgery: principles and techniques. 4th ed. Massachusetts: Blackwell; 1996. p. 195-208.

53. Keller PK, Silver D. Anticoagulants: heparin and warfarin. In: Vieth FJ, Hobson RW, Williams RA, Wilson SE. Vascular surgery: principles and practice. 2nd. ed. New York: McGraw-Hill; 1994; p. 219-27.

54. Bovill EG, Becker R, Tracy RP. Monitoring thrombolytic therapy. Prog Cardiovasc Dis. 1992;34:279-94.

55. Estelles A, Aznar J, Gilabert J, Parrilla JJ. Dysfunctional plasminogen in full-term newborn. Pediatr Res. 1980;14:1180-5.

56. Marder VJ, Sherry S. Thrombolytic therapy: current status (1). N Engl J Med. 1988;318:1512-20.

57. Marder VJ, Sherry S. Thrombolytic therapy: current status (2). N Engl J Med. 1988;318:1585-95.

58. Reznik VM, Anderson J, Griswold WR, Segall ML, Murphy JL, Mendoza SA. Successful fibrinolytic treatment of arterial thrombosis and hypertension in a cocaine-exposed neonate. Pediatrics. 1989;84:735-8.

59. Zenz W, Arlt F, Sodia S, Berghold A. Intracerebral hemorrhage during fibrinolytic therapy in children: a review of the literature of the last thirty years. Semin Thromb Hemost. 1997;23:321-32.

Correspondência:

Nilo César Barbosa Mandelli

Dona Laura, 354/401, Rio Branco

CEP 90430-090 - Porto Alegre, RS

Tel.: (51) 3331.4477 (51) 3024.5480

E-mail: ncbm@terra.com.br 